\title{
Proportion of Intestinal Obstruction and Associated Factors Among Patients with non Traumatic Acute Abdomen Admitted to Surgical Ward in Debre Birhan Referral Hospital, North East Ethiopia
}

\author{
Mesfin Yohannes ${ }^{1,}$,, Muluken Fanta ${ }^{1}$, Tesfahun Molla ${ }^{2}$ \\ ${ }^{1}$ Wolaita Zone Health Department, Southern Nations Nationalities and People Region State, Wolaita Sodo, Ethiopia \\ ${ }^{2}$ School of Medicine, Collegeof Health Sciences and Medicine, Wolaita Sodo University, Wolaita Sodo, Ethiopia \\ Email address: \\ messyohan11@gmail.com (M. Yohannes),mulerhofa@gmail.com (M. Fanta), tesfamolla@gmail.com (T. Molla) \\ ${ }^{*}$ Corresponding author
}

\section{To cite this article:}

Mesfin Yohannes, Muluken Fanta, Tesfahun Molla. Proportion of Intestinal Obstruction and Associated Factors Among Patients with non Traumatic Acute Abdomen Admitted to Surgical Ward in Debre Birhan Referral Hospital, North East Ethiopia. American Journal of Biomedical and Life Sciences. Vol. 5, No. 3, 2017, pp. 54-62. doi: 10.11648/j.ajbls.20170503.13

Received: March 24, 2017; Accepted: April 5, 2017; Published: June 28, 2017

\begin{abstract}
Intestinal obstruction (IO) continues to be a major problem for human being since it was first recognized and treated in 350 Before Christ. Different treatment options were developed since then with only minimal reduction in morbidty and mortality. It occurs when the normal flow of intestinal content is interrupted. Intestinal obstruction is the most common causes of emergency surgical admission through out world. It is also one of the major causes of morbidity and mortalities associated with acute abdomen. The proportion and etiologies of intestinal obstruction differ not only from country to country but also with in the different regions of the same country. Knowing proportion of obstruction is very important to manage patients accordingly. Only very few data concerning proportion of intestinal obstruction is found in Ethiopia and none in Debre Birhan. The aim of the study is to assess the proportion of intestinal obstruction and associated factors among patients with non traumatic acute abdomen admitted to surgical ward in Debre Birhan Referral Hospital from December 8,2015 to January 9,2016 . Facility based cross-sectional study was conducted by using 357 patient's record with acute abdomen from December 8,2015 to January 9,2016. Collected data was entered in to SPSS version 20. Adjusted Odds ratio with $95 \%$ confidence interval and p-value were used to measure associations. From 357 study subjects $181(50.7 \%)$ and $141(39.5 \%)$ were Intestinal obistruction and acute appendicitis cases respectively with response rate of $99.4 \%$. large bowel obstruction (LBO) accounts for about $109(60.2 \%)$ of cases while $72(39.8 \%)$ of cases were small bowel obstruction (SBO). Male gender, residence and constipation are significantly associated with intestinal obstruction $(\mathrm{P}<0.05)$. From all study subjects those who had previous history of constipation were $61(33.7 \%)$, previous history of abdominopelvic operation $25(7 \%)$, previous history of intestinal obstruction $35(9.8 \%)$, hernia $12(3.4 \%)$ and previous history of rectal bleeding $5(1.4 \%)$. Intestinal obstruction is the most common cause for non traumatic acute abdominal admission accounting $50.7 \%$. In the study area Male gender, constipation and urban residence are significantly associated with intestinal obstruction. Postoperative adhesion and garoin hernias were found to be the commonest causes for intestinal obstruction next to Volvulus, but tumor was the rare cause of small bowel obstruction in the study area. Elective repair of hernia should be strengthened before it causes obstruction. Public awareness should be increased on the risk factors of intestinal obstruction through health education to reduce the observed magnitude. Moreover, health facilities and health professionals capable of handling patients with intestinal obstruction should be available with in the reach of the community.
\end{abstract}

Keywords: Intestinal Obstruction, Small Bowel Obstruction, Large Bowel Obstruction, Hernia 


\section{Introduction}

\subsection{Background}

Intestinal obstruction occurs when the normal flow of intestinal contents is interrupted. Intestinal obstruction is one of the most common causes of acute abdomen world wide. It was recognized, described, and treated by Hippocrates (460370 Before Christ.). At that time it was believed that living for God than for man is better if patients with Intestinal obstruction have reached stage of bowel perforation. At that time had ileus developed in consequence of Intestinal obstruction, the mortality rate reached $100 \%$. Both surgical and conservative treatment options were tried since 350 Before Christ, but success rate of both treatments was minimal until the $20^{\text {th }}$ century. Surgical (Protagoras, 350 Before Christ., enterocutaneous fistula) and conservative (reposition of incarcerated hernias, analgesic like opium, ingestion of heavy metals like mercury or lead and leeches to remove toxic agents from the blood) were among documented treatment options at that time [1].

An improvement took place after 1912, when Hartwell and Hoguet noticed that infusion of the physiological solution prolonged the survival of dogs with Intestinal obstruction. The second chief therapeutic principle-suction by nasogastric or intestinal tubes was gradually introduced into clinical practice in the 1920's (McIver, 1926). Antibiotics began to represent a standard part of therapy algorithms of intestinal obstruction in the 1940' and 1950's. The introduction of this management options reduced mortality rate from $50 \%$ in 1930 s to about $10 \%$ in 1960 s [2].

The pessimism of leaving for God, if the patient presents with perforated intestine, are now gradually disappeared as safe techniques evolved for intestinal suturing and anstomosis. Infusion therapy, intestinal suction, antibiotics, somatostatin, new imaging methods like ultra sonography and computed tomography scanning, together with improved surgical and anesthetic techniques has substantially reduced the mortality rate [3].

The most common cause of Intestinal obstruction is adhesion in developed countries but is either Volvulus or hernia in most African countries. It is common to see patients with huge groin hernias which grows down to their scrotum or thigh and presents late after they developed Intestinal obstruction which is of course gangrenous in majority of cases and hence carries high risk of morbidity and mortality in Ethiopia and Debre Birhan Referral Hospital in particular. It is also common to see an old man with grossly distended abdomen from sigmoid volvulus seeking tube decompression or coming so late after intestinal ischemia has occurred needing colostomy (artificialintestinalopeningtheabdomen) in Debre Birhan Referral Hospital [4].

\subsection{Conceptual Frame Work}

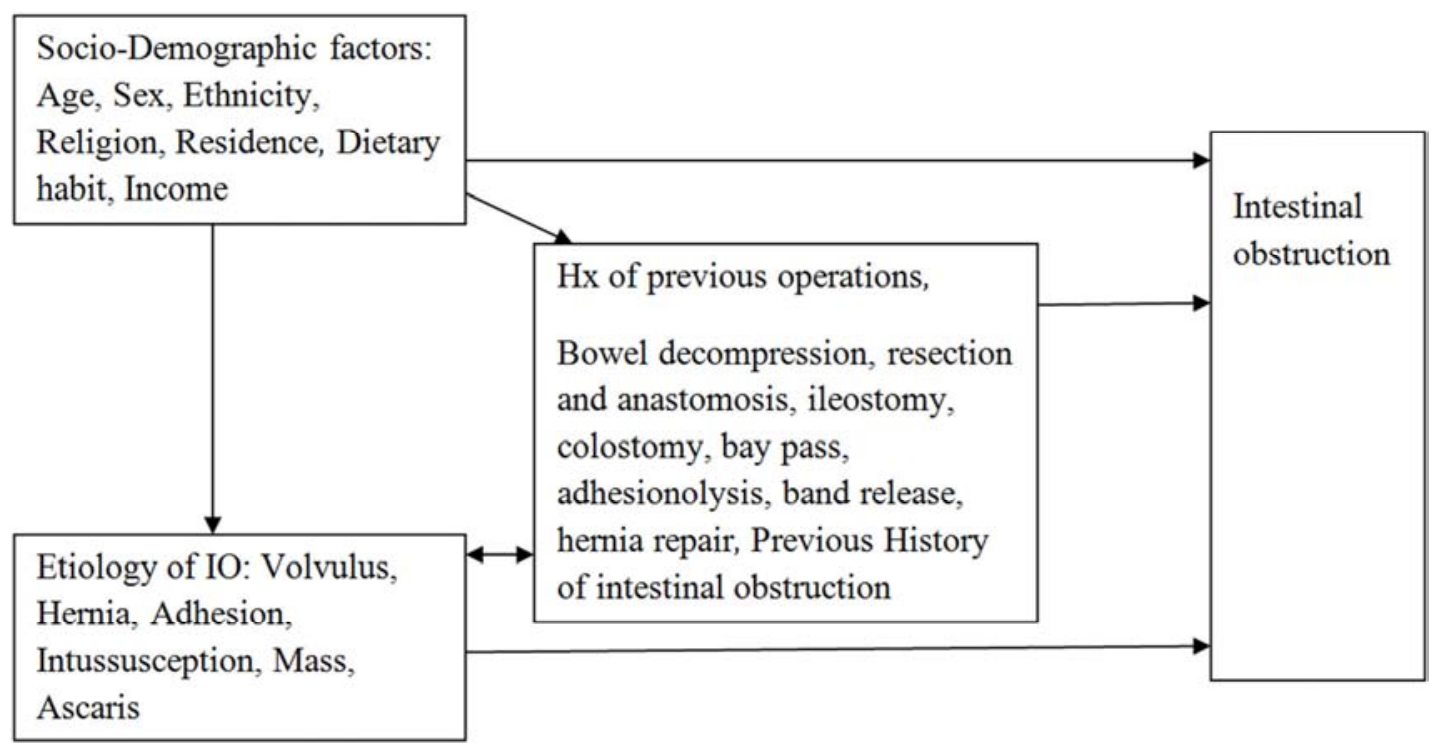

Figure 1. Conceptual frame work on intestinal obstruction modified from different literatures.

\section{Methods and Materials}

\subsection{Study Area}

The study was conducted at Debre Birhan referral hospital which is located in Debre Birhan town, $135 \mathrm{kms}$ away from Addis Ababa and $695 \mathrm{kms}$ from the capital city of the region,
Bahir Dar. The hospitals catchment total population is estimated to be 2,400,000 with females $50 \%$. There are one private hospital, three health centers, twenty private clinics, twelve private pharmacies, one health science college and one university. The Debre Birhan Referral Hospital is staffed with 4 general surgeon, 2 gynecologist and obstetrician, 1 internist, 1 pediatrician, 4 emergency surgical professional 
specialist, 24 general practitioners, 2 dental physicians, 2 Psychiatry Msc, 1 health officer, 147 nurses, 22 midwives, 231 aboratory proffesionals, 23 pharmacy professionals, 10 anesthetist, 2 enviromental health professional, 1 health education professional, $5 \mathrm{x}$-ray profesinals, 2 ophtometric and 2 physiotherapist. Totally the hospital has 121 beds. The department of Surgery has atotal of 31 beds.

\subsection{Study Design and Period}

Facility based cross-sectional study was conducted from December 8,2015 to January 9,2016.

\subsection{Source Population}

All non traumatic acute abdominal cases.

\subsection{Study Population}

All non traumatic acute abdominal cases who were admitted to surgical ward of Debre Birhan Referral Hospital from July 8,2014 to June 8,2015.

\subsection{Inclusion and Exclusion Criteria}

\subsubsection{Inclusion Criteria}

All patients with diagnosis of non traumatic acute abdomen cases who were admitted to surgical ward from July 8,2014 to June 8,2015 were included.

\subsubsection{Exclusion Criteria}

Patient went home against medical adivice before completion of treatment (data on final outcome can't be obtained) were excluded.

\subsection{Sample Size Determination}

A study conducted in black lion hospital by B.Kotiso and Z.Abdurahman showed that the proportion of patients with intestinal obstruction among all non-traumatic cases of acute abdomen is $26 \%$ [12].

Confidence level of $95 \%$ is specified.

Margin of error was 5\%. Therefore:-

(1) $n=Z^{2 \frac{\alpha}{2}}\left(\frac{p(1-P)}{d^{2}}\right)$

Where $\mathrm{n}=$ samplesize

$\mathrm{P}=0.26$ (proportion of patients with intestinal obstruction) $\mathrm{d}=$ marginal error between sample and population $(0.05)$

$95 \%$ confidence interval.

(2) $\mathrm{n}=1.96 * 1.96 * .26 * .74 / 0.05 * 0.05=297$

The total sample size was $=297$ But, only 1,711 acute abdomen cases were found at the hospital during study period.

We used correction to get final sample as follows.

Let say $-\mathrm{n}_{\mathrm{o}}$ intial sample size which is 297 .

$-\mathrm{N}=$ is finite population size which is 1,711

$-n=$ is final sample size to be calculated $=$ ?

(3) $\left.\mathrm{n}=\mathrm{n}_{\mathrm{o}} /\left(1+\left(\mathrm{n}_{\mathrm{o}} / \mathrm{N}\right)\right)=297 /(1+297 / 1711)\right)=254$

Considering non response rate of $10 \%, \mathrm{n}=281$

The above sample size is for first specific objective, but to check whether the above sample size is sufficient to asses associated factors for intestinal obstruction, sample size can be calculated for some independent variables using Ep iinfo statistical program. Assumptions are taken as confidence level $=95 \%$, power $=80 \%$, Unexposed: Exposed ratio 1:1, odds ratio $=2$

Table 1. Total number of sample size by assuming confidence level $=95 \%$, power $=80 \%$, Unexposed: Expose dratio $: 1$, odds ratio $=2$ ).

\begin{tabular}{|c|c|c|}
\hline Determinant factors & Assumptions (confidence level $=95 \%$, power $=80 \%$, Unexposed: Exposed ratio1:1,odds ratio $=2$ ) & Sample size \\
\hline \multirow{3}{*}{ Postopadhesion } & \multirow{3}{*}{ Proportion of exposure among intestinal obstruction (51.2\%) (source (13) } & Exposed $=128$ \\
\hline & & unexposed $=128$ \\
\hline & & Total $=296$ \\
\hline \multirow{3}{*}{ Sigmoid volvulus } & \multirow{3}{*}{ Proportion of exposure among intestinal obstruction $(58.6 \%)$ (source(13) } & Exposed $=162$ \\
\hline & & unexposed $=162$ \\
\hline & & Total $=324$ \\
\hline
\end{tabular}

Therefore, the total number of sample size $n=324$. Considering non response rate of $10 \%, \mathrm{n}=357$.

\subsection{Sampling Procedure}

Medical record numbers were sorted from smallest to large stand coded from 1-1711. Required sample size was obtained by using simple random sampling technique. Selected charts were collected from the card room and presence of required information was checked and 3 Charts with very few information were replaced with 3 other charts from previously not selected charts by lottery method before data was filled in data collection tool.

\subsection{Variables}

\subsubsection{Dependent Variable}

Intestinal obstruction (Yes, No)

\subsubsection{Independent Variables}

a. Socio-Demographic characteristics

Age

Sex

Residence

Ethnicity

Religion

b. Patient history

History of Previous operation.

Previous History of intestinal obstruction.

History of rectal bleeding

History of constipation

c. Physical examination

Presence of abdominal wall hernia 


\subsection{Data Collection Procedures}

Data was collected by using structured data extraction format which was prepared in English language. The data extraction format was pre-tested on 13 patient charts in Debre Birhan Hospital. Vague questions and other simila rmistakes were corrected before the actual data collectionis begun. The actual data collection wass tarted after one day of training on data extraction format was given to data collectors. In the training session, the data collectors' were oriented on the objectives of the study, how to collect data and keep confidentiality of information obtained. The principal investigator was the leader of the data collection and checks daily the completeness and consistency of the data after collection.

\subsubsection{Data Quality Control}

To keep the quality of data, training was given for data collectors. Day to day activities during data collection was supervised and evaluated. Errors found were corrected by data collectors before the following day activity.

\subsubsection{Data Entry and Cleaning}

After data collection, each questionnaire was given a unique code by the principal Investigator. The principal investigator entered data to SPSS version 20 statistical software. Frequencies were used to check for missed values. Any errors identified at this time were corrected after revision of the original data using the code numbers.

\subsubsection{Data Analysis}

Frequencies and Proportions were used for description of the study population in relation to socio-demographic and other relevant variables. Binary logistics regression analysis was initially perfomed to assess the presence of association between independent and dependent variables. Multivariate logestic regression was then performed to control potential confounding variables. The strength of association between independent and dependent variables were assessed using adjusted odds ratio with $95 \%$ confidence interval and pvalue.

\subsection{Standard Procedures}

Bowel anstomosis:-it is the type of suturing two parts of gastrointestinal system together after resecting diseased part or to biapass the diseased part in between. There are different types of bowel anstomosis based on the pathology. It can be end-to-end, end-to-side or side-to-end.

Bowel decompression:-it is the type of procedure to empty or to relieve the distended or obstructed intestine by either inserting nasogastric tube or rectal tube. It can also be done intraopertively by milking the intestinal content either retrogradly towards stomach and then sacked out by anesthetist by NG tube or antigradly towards large intestine and then out through rectal tube. Rarely can it be done by making small opening on the antimesentric border of intestine, but it is a futile exercise and not usually recommended.

Bowel derotation:-is a procedure in which intestine is derotated or detwisted in its Owen mesenteric axis back in to its normal position as in case of small bowel volvulus or sigmoid volvulus after doing laparatomy and confirming that the bowel is viable.

Bowel Resection:-is procedure in which parts of intestine which is considered to be non functional or non viable is cut and removed surgically.

Colostomy:-The opening of a portion of the large intestine (colon) through the abdominal wall to its skin surface. This procedure is usually performed in patients with gangrenous large bowel obstruction, traumatic perforation of large intestine, to bypass distal anstomosis and other similar procedures.

Hartmann's Procedure:- It refers to a colon or rectal resection without an anstomosis in which a colostomy or ileostomy is created and the distal colon or rectum is left as a blind pouch. The term typically is used when the left or sigmoid colon is resected and the closed off rectum is left in the pelvis. If the distal colon is long enough to reach the abdominal wall, a mucus fistula can be created by opening the defunctioned bowel and suturing it to thes kin.

Herniorrhaphy:-is a procedure which involves legating the neck of hernia followed by cutting the sac (Herniotomy) and repair of the defect through which the sac has protruded. This is the procedure of choice in adults to reduce the risk of future recurrence.

Herniotomy:-is a procedure which involves legating the neck of hernia followed by cutting the sac. This procedure is usually done in infants and children with congenital hernia.

Ileostomy:-A surgical opening in the abdominal wall, through which a segment of ileum is exteriorized through anterior abdominal wall.

Laparotomy:-is surgically entering in to peritoneal cavity by making one of the different incisions. The type of incision depends on site of pathology, type of procedure, experience of surgeon, diagnostic uncertainty and urgency. Based on this factors, incision can be midline (longmidline, supraumblical, impraumblical or indeterminate), transverse and oblique.

Nasogastrictubedecompression:-A long tube inserted through the nose into the stomach for decompression and its tip can be advancement into the small Intestine. The most common use is to relieve the abdominal distention associated with intestinal obstruction.

Rectaltubedefilation:-Rigid or flexible tube is inserted through anus and into rectum to empty its contents. This procedure is usually done to relieve large bowel obstruction from sigmoid volvulus.

Relaprotomy:-it is operating the same patient for the second or more times. It can be done either due to complication of previous operation or to perform remaing procedures after previous damage control surgery.

Totalcolectomy:-it is surgical excision of colon (cecum, ascending, transverse, descending and sigmoid colon) with either iliorectal anstomosis or permanent ileostomy. 
Total Proctocolectomy:-In this procedure, the entire colon, rectum, and anus are removed and the ileum is brought to the skin as a Brooke ileostomy.

\section{Results}

\subsection{SocioDemographic Factors}

Larger proportion of study subjects 205(57.4\%) were males with response rate of $99.4 \%$. The age of patients ranged from 2 months to 88 years, of which $93(26.1 \%)$, 92(25.8\%), 82(23\%), 70(19.6\%), 14(3.9\%) and 6(1.7\%) were in the age range $5-14,>60,15-40,40-60,1-5$ and less than 1 year respectively.

One hundred thirty two $(37 \%)$ of patients were from Debre Birhan town while 225(63\%) of patients were from outside Debre Birhan Town. Majority of patients 214(59.9\%), belong to Amhara ethnic group followed by Oromo 134(37.6\%), Tigrie $5(1.4 \%)$ and $4(1.1 \%)$ were others.

About 257(72\%) were Orthodox and 84(23.5\%) and 11(3.1\%) were Muslims and Protestants by religion respectively. (SeeTable2)

\subsection{Clinical Findings}

Abdominal pain 341(95.5\%), abdominal distension $220(61.6 \%)$ and Naussea and vomiting 306(85.7\%) are the three most frequent history findings. Failure to pass feces 254(71.1\%), failure etopass flatus 220(61.6\%), Previous history of constipation $72(20.2 \%)$, previous history of abdominopelvic operation $25(7 \%)$, previous history of intestinal obstruction $35(9.8 \%)$, hernia $12(3.4 \%)$ and previous history of rectal bleeding $5(1.4 \%)$ are other history findings in the study subjects. From total cases $113(31.7 \%)$ presented to hospital within 24 hours and other $122(34.2 \%)$ of presented with in 24-48 hours of onset of illness while $69(19.3 \%)$ and $53(14.8 \%)$ of patients presented with in $48-72$ hours and after 72 hours respectively. (Seetable 3 )

Blood pressure (BP) was not recoded (record not found) during data collection time in $41(12.1 \%)$ of cases. From patients whose BP recorded $29(8.1 \%)$ are hypotensive (systolic $\mathrm{BP}<90$ ) and $17(4.8 \%$ ) are hypertensive (systolic $\mathrm{BP}>140)$. In the remaining $270(75.6 \%)$ of cases $\mathrm{BP}$ is in normal range. Pulse rate is normal (60-99) in $319(89.4 \%)$ while it is elevated in remaining $38(10.6 \%)$ of cases. Fever (temperature $>37.5$ ) is seen in $124(34.7 \%$ ) and in $41(11.5)$ of cases low tempreture $(<36)$ is recorded. Temprture record was found in normal range for $192(53.8 \%)$ of patients. Respiratory rate is recorded for all (100\%) cases with $164(45.9 \%)$ are in normal range with remaining $150(42 \%)$ and $43(12)$ are having tachypenia $(\mathrm{RR}>20)$ and bradypenia $(\mathrm{RR}<16)$. From recorded cases $140(39.2 \%)$ and $212(59.4 \%)$ of $\mathrm{WBC}$ and hematocrit are with in normal range ( $\mathrm{WBC}=4$ 11 and $\mathrm{HCT}>36$ ) respectively.

Abdominal examination revealed hypoactive and hyperactive bowel sound in 157(44\%) and 190(53.2\%) of cases respectively. Abdomen was soft in 140(39.2\%), direct tenderness in $46(12.9 \%)$ and rebound tenderness in
158(44.3\%). Per rectal examination results showed full of stool, scantys tool, empty rectum and bleeding in the examining finger in $153(42.9 \%), 82(23 \%), 110(30.8 \%)$ and $1(0.3 \%)$ of cases respectively.

Table 2. Sociodemographic characitericitics of patients with acute abdomen at Debre Birhan Referral Hoapital 2014/15(N=357).

\begin{tabular}{llll}
\hline Variables & & Number & $\mathbf{\%}$ \\
\hline \multirow{2}{*}{ Sex } & Male & 205 & 57.4 \\
& Female & 152 & 42.6 \\
& $0-11$ month & 6 & 1.7 \\
Age in years & $1-5$ & 14 & 3.9 \\
& $5-14$ & 93 & 26.1 \\
& $15-40$ & 82 & 23.0 \\
Residence & $40-60$ & 70 & 19.6 \\
& $>60$ & 92 & 25.8 \\
& Debre Birhan & 132 & 37 \\
Ethnicity & Outside Debre Birhan & 225 & 63 \\
& Amhara & 214 & 59.9 \\
& Oromo & 134 & 37.6 \\
& Tigrie & 5 & 1.4 \\
Religion & Others & 4 & 1.1 \\
& Orthodox & 257 & 72 \\
& Muslim & 84 & 23.5 \\
& Protestant & 11 & 3.1 \\
& Others & \\
\hline
\end{tabular}

${ }^{1}$ Afar $=4 ; \quad{ }^{2}$ Catholic $=5$

Table 3. Frequency table of history findings of patients with acute abdomen at Debre Birhan Referral Hoapital 2014/15 $(N=357)$.

\begin{tabular}{|c|c|c|c|}
\hline Variables & & Number & $\%$ \\
\hline \multirow{2}{*}{ History of abdominal pain } & YES & 341 & 95.5 \\
\hline & $\mathrm{NO}$ & 16 & 4.5 \\
\hline \multirow{2}{*}{ Nausea and vomiting } & YES & 306 & 85.7 \\
\hline & NO & 51 & 14.3 \\
\hline \multirow{2}{*}{ Failure to pass feces } & YES & 254 & 71.1 \\
\hline & NO & 103 & 28.9 \\
\hline \multirow{2}{*}{ Failure to pass flutus } & YES & 220 & 61.6 \\
\hline & NO & 137 & 38.4 \\
\hline \multirow{4}{*}{ Duration of symptoms in hours } & $<24$ & 113 & 31.7 \\
\hline & $24-47$ & 122 & 34.2 \\
\hline & $48-72$ & 69 & 19.3 \\
\hline & $>72$ & 53 & 14.8 \\
\hline \multirow{2}{*}{ Abdominal distention } & YES & 220 & 61.6 \\
\hline & NO & 137 & 38.4 \\
\hline \multirow{2}{*}{ History of constipation } & YES & 72 & 20.2 \\
\hline & NO & 285 & 79.8 \\
\hline \multirow{2}{*}{ History of diarrhea } & YES & 8 & 2.2 \\
\hline & NO & 349 & 97.8 \\
\hline \multirow{2}{*}{ History of bleeding per anus } & YES & 5 & 1.4 \\
\hline & NO & 352 & 98.6 \\
\hline \multirow{2}{*}{$\begin{array}{l}\text { History of previous abdominal or pelvic } \\
\text { operation }\end{array}$} & YES & 25 & 7 \\
\hline & NO & 332 & 93 \\
\hline \multirow{2}{*}{ Previous history of intestinal obstruction } & YES & 35 & 9.8 \\
\hline & NO & 322 & 90.2 \\
\hline \multirow{2}{*}{ Hernia } & YES & 12 & 3.4 \\
\hline & NO & 345 & 96.6 \\
\hline
\end{tabular}

\subsection{Proportion of Intestinal Obstruction}

From study subjects $181(50.7 \%)$ cases were intestinal obstruction. From the total cases of obstruction $72(39.8 \%)$ are small bowel obstruction and 109(60.2\%) are large bowel obstruction with the remaining $141(39.5 \%), 15(4.2 \%), 11(3.1 \%)$, $2(0.6 \%), 3(0.8 \%), 2(0.6 \%), 1(0.3 \%)$, and $1(0.3 \%)$ cases were due 
to acute appendicitis. cholecystitis, Perforated peptic ulcer disease(PPUD), ilialperforation, Pelvic inflammatory Disease(PID), primary peritonitis, intraabdominal abscess and pancreatitis respectively. From small bowel obstruction $57(79.2 \%)$ cases are simple and $15(20.8 \%)$ are gangrenous small bowel obstruction. From the large bowel obstruction cases 97(89\%) are simple and $12(11 \%)$ are gangrenous.

The commonest causes detected during preoperative period are Sigmoid volvulus1 05(58\%), Small bowel volvulus $36(19.9 \%)$, Adhesion $15(8.3 \%)$, Intussusception $8(4.4 \%)$ and Hernia1 2(6.6\%). From this data, I can say that the most common cause of intestinal obstruction is sigmoid volvulus. The most common causes of small bowel obstruction is Small bowel volvulus .It is the second most common cause of intestinal obstruction. Adhesion, hernia and intussusceptions are the third, fourth and fifth most common cause of intestinal obstruction in general and the second, third and fourth most common causes of small bowel obstruction in particular. Tumor [5] and a ganglionic megacolon [1] are rare causes of large bowel obstruction. From operated case of intestinal obstruction sigmoid volvulus was found in 45 cases while Small bowel volvulus, hernia, adhesion, intussusceptions and tumor are found to be the cause in $25,12,11,8$ and 5 respectively.

Table 4. Proportion of intestinal obstruction and associated factors among admitted patients at Debre Birhan Referral Hospital 2014/15 $(N=181)$.

\begin{tabular}{llll}
\hline Variable & & Number & \% \\
\hline & SBO & 72 & 20.2 \\
& LBO & 109 & 30.5 \\
& Appendicitis & 141 & 39.5 \\
& PPUD & 11 & 3.1 \\
& Cholecystittis & 15 & 4.2 \\
Types of acute abdomen & PID & 3 & 0.8 \\
cases & Ileal perforation & 2 & 0.6 \\
& Primary peritonitis & 2 & 0.6 \\
& Intra abdominal & 1 & 0.3 \\
& abscess & 1 & 0.3 \\
& Pancreatitis & 57 & 16 \\
& Simple SBO & 15 & 4.2 \\
Clinical classification & Gangrenous SBO & 97 & 27.2 \\
before operation & Simple LBO & 12 & 3.4 \\
& Gangrenous LBO & 38 & 10.6 \\
Clinical classification of & Simple SBO & 15 & 4.2 \\
intestinal obstruction (from & Gangrenous SBO & 33 & 9.2 \\
operated cases) & Simple LBO & 16 & 4.5 \\
\hline
\end{tabular}

\begin{tabular}{llll}
\hline Variable & & Number & \% \\
\hline & Sigmoid volvulus & 105 & 29.4 \\
& Hernia & 12 & 3.4 \\
Etiology of obstruction & Adhesion & 15 & 4.2 \\
Before operation & Small bowel volvulus & 36 & 10.1 \\
& Tumor & 5 & 1.4 \\
& Intussusception & 8 & 2.2 \\
& Sigmoid volvulus & 45 & 12.6 \\
& Hernia & 12 & 3.4 \\
Etiology of obstruction & Adhesion & 11 & 3.1 \\
(from operated cases) & Small bowel volvulus & 25 & 7 \\
& Tumor & 5 & 1.4 \\
& Intussusception & 8 & 2.2 \\
\hline
\end{tabular}

\subsection{Treatment of Intestinal Obstruction}

From all 181 cases of intestinal obstruction 73(40.3\%) and $108(59.7 \%)$ are treated conservatively and surgically respectively. Majority of conservatively managed cases are large bowel obstructions accounting for about $64(17.9 \%)$ and the remaining $9(2.5 \%)$ are small bowel obstruction cases. Rectal tube defilation is used in all 64 of simple large bowel obstruction cases while $\mathrm{Ng}$ tube decompration is used in all 9 of small bowel obstruction cases. This shows that conservative treatement most commonly used in large bowel obstruction cases when compered to small bowel obstruction cases. From 108 operated cases 56 (51.9\%) are small bowel obstruction cases. From this data it is clear that majority of small bowel obstruction cases are managed by surgery, but majority of large bowel obstruction cases are managed conservatively.

Failed conservative management, Gangrenous small bowel obstruction, Gangrenous large bowel obstruction and strangulated hernia are the four most commonly stated reason for operation accounting 69(19.3\%), 11(3.1\%), 16(4.5\%) and $12(3.4 \%)$ of operatedcases.

Bowel derotation and decompression 43(39.8\%), bowel resection and anstomosis 16(14.8\%), Colostomy 16(14.8\%), hernial operation $12(11.1 \%)$, reduction of intusuception $8(7.4 \%)$, bowel perforation repair $2(1.9 \%)$ release of band and adhesion $11(10.2 \%)$ are the operations (procedures) performed during operation.

All 108 operated cases took preoperative antibiotics and 4 of operated cases transfused with blood.

Table 5. Frequency tabl e of treatment types and procedure in patients with intestinal obstruction admitted to Debre Birhan Referral Hospital 2014/15(N=181).

\begin{tabular}{|c|c|c|c|}
\hline Variable & & Number & $\%$ \\
\hline \multirow{2}{*}{ Treatment types for intestinal obstruction } & Conservative & 73 & 40.3 \\
\hline & Surgical & 108 & 59.7 \\
\hline \multirow{3}{*}{ Conservative treatments } & $\mathrm{Ng}$ tube + iv fluid & 8 & 4.4 \\
\hline & Rectal tube + iv fluid & 63 & 34.8 \\
\hline & Others $^{3}$ & 2 & 1.1 \\
\hline \multirow{4}{*}{ Reason for surgery } & Failed conservative management & 54 & 29.8 \\
\hline & Strangulated hernia & 12 & 6.6 \\
\hline & Gangrenous LBO & 16 & 8.8 \\
\hline & Others ${ }^{4}$ & 15 & 8.3 \\
\hline Procedues done & Derotation and dedecompression & 44 & 24.3 \\
\hline
\end{tabular}




\begin{tabular}{llll}
\hline Variable & & Number & \% \\
\hline & Bowel peforation repair & 2 & 1.1 \\
& Bowel resection and colostomy & 16 & 8.8 \\
& Bowel resection and primary anstomosis & 15 & 8.3 \\
& Reduction of intussusceptions & 8 & 4.4 \\
& Release of band and adhesion & 11 & 6.1 \\
Antibiotics given & Hernial operation & 12 & 6.6 \\
& Yes & 131 & 36.7 \\
Blood transfused & No & 50 & 14 \\
& Yes & 4 & 2.2 \\
\hline
\end{tabular}

3Enema; 4SBV=9, Ascuris=3, HPS=2andaganglionicmegacolon=1

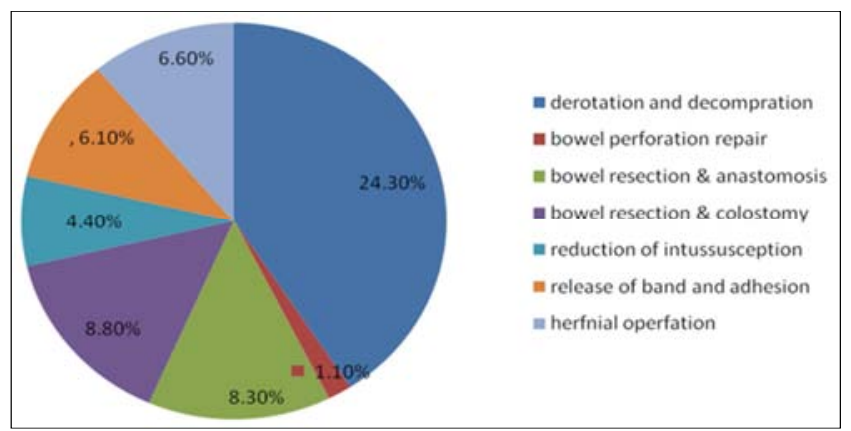

Figure 2. Piechart of different surgical procedures of patients with intestinal obstruction at Debre Birhan Referral Hospital 2014/15.

\subsection{Out Comes of Treatment}

Post operative complication were seen in $18(16.7 \%)$ of 108 operated cases making overall complication rates of about $9.9 \%$. The most frequently observed complication is postoperative wound infection which is seen in 14(12.9\%) of all operated cases. The other complications include anastomotic leakage in $1(0.9 \%)$, wound dehiscence in $2(1.8 \%)$, and pneumonia in one $(0.9 \%)$ of case. Relaparatomy is performed in $3(2.7 \%)$ operated cases with anastomotic leakage $1(33.3 \%)$ and wound dehiscence $2(66.7 \%)$ each are the reasons for second operation. From these three reoperated patients one died after second operation making death rate after relaparatomy $33.3 \%$. Total number of days patients stayed in Hospital range from 119days.

The over all success rate of treatement of acute intestinal obstruction is $98.3 \%$ with 181 of the study subjects improved discharged and $3(1.6 \%)$ of cases died. Non of patient died during conservative treatement. All 3 deaths occurred after operative treatment with 2 occurred after first operation and 1 occurred after second operation. Death rate after second operation is high with $1(50 \%)$ of 2 reoperated cases died when compered to $2(1.9 \%)$ of 107 cases who died after first operation. This implies death rate is high if the patient is reoperated during the same admission. Infectin and sepsis were causes of death while fluid and electrolyte imbalance (shock) in 1 (seetable 6)

Table 6. Frequency table of treatement out come of patients with intestinal obstruction at Debre Birhan Referral Hospital 2014/15 (N=181).

\begin{tabular}{llll}
\hline Variables & & frequency & \% \\
\hline \multirow{2}{*}{ Post operative } & Wound infection & 14 & 12.9 \\
complication & Leakage & 1 & 0.9 \\
& Others $^{5}$ & 3 & 2.8 \\
Relapartomy done & Yes & 3 & 2.8 \\
Reason for & No & 105 & 97.2 \\
relapartomy & Leakage & 1 & 0.9 \\
Treatment outcome & Wound dehiscence & 2 & 1.8 \\
Possible cause of & Improved and discharged & 105 & 97.2 \\
death? & Died & 3 & 2.8 \\
\hline
\end{tabular}

${ }^{1}$ Pneumonia $=1$ andwounddehiscence $=2$

\subsection{Bivariate and Multivariate Analysis}

From the independent variables sex, residence and history of chronic constipation are significantly associated with intestinal obstruction. After cheking for the presence of association $(p<0.25)$ in binary logestic regression, this variables werere-entered into multivariate logestic regression to assess the strength of association between this variables and intestinal obstruction by controlling the confounding effect of other variables.

Being male sex is arisk for intestinal obstruction $(p=0.001)$ and adjusted odds ratio (AOR) of 3.048 and $95 \%$ CI [1.5745.903]. From the above statement it is clear that males are 3 times more likely to develop intestinal obstruction than females.

Urban residence is also significantly associated with intestinal obstruction with $\mathrm{p}<0.001, \mathrm{AOR}=21.355$ and $\mathrm{CI}$ [9.6-47.4].

History of chronic constipation is also a risk for intestinal obstruction $(\mathrm{p}<0.001), \mathrm{AOR}=13.729$ and CI [5.542-34.009]. From thiss tatement it is clear that having constipation is 13.7 times more likely to develop intestinal obstruction. (Seetable 7)

Table 7. Bivariate and multivariate analysis of patients with intestinal obstruction at Debre Birhan Referral Hospital $2014 / 15$.

\begin{tabular}{|c|c|c|c|c|c|}
\hline \multirow{2}{*}{ Variables } & \multicolumn{2}{|l|}{ IO } & \multirow{2}{*}{$\operatorname{COR}(95 \% \mathrm{CI})$} & \multirow{2}{*}{$\operatorname{AOR}(95 \% C I)$} & \multirow{2}{*}{ p-value } \\
\hline & Yes & No & & & \\
\hline \multicolumn{6}{|l|}{ Sex } \\
\hline Male & 86 & 119 & $2.054(1.340,3.150)$ & $3.048(0.1 .574,5.903)$ & .001 \\
\hline Female & 95 & 57 & 1.00 & 1.00 & \\
\hline Residence & & & & & $<0.001$ \\
\hline
\end{tabular}




\begin{tabular}{|c|c|c|c|c|c|}
\hline \multirow{2}{*}{ Variables } & \multicolumn{2}{|l|}{ IO } & \multirow[b]{2}{*}{$\operatorname{COR}(95 \% \mathrm{CI})$} & \multirow{2}{*}{$\operatorname{AOR}(95 \% C I)$} & \multirow{2}{*}{ p-value } \\
\hline & Yes & No & & & \\
\hline Debre Birhan & 17 & 115 & $18.603(10 . .325,33.519)$ & $21.355(9.618,47.414)$ & \\
\hline Outside Debre Birhan & 164 & 61 & 1.00 & 1.00 & \\
\hline \multicolumn{6}{|l|}{ History of contipation } \\
\hline Yes & 61 & 11 & $7.625(3.794,14.890)$ & $13.729(5.542,34.009)$ & $<0.001$ \\
\hline No & 120 & 165 & 1.00 & 1.00 & \\
\hline
\end{tabular}

\section{Discussion}

The leading causes of acute abdomen in the study area are intestinal obstruction $181(50.7 \%)$ and acute appendicitis in $141(39.5 \%)$ of cases. Majority of intestinal obstruction cases $60.2 \%$ are large bowel obstruction. Althouhg this finding are similar to other studies in our country and other African country, it is different from recent study done in Black Lion Hospital which has shown that the most common cause of acute abdomen was acute appendicitis and the most common type of intestinal obstruction is small intestinal obstruction [3 $4,5,7]$. This finding is also different from the global reference in which $80 \%$ of intestinal obstruction is due to small bowel obstruction and the leading cause for acute abdominal admission are due to acute appendicitis [9]. Majority $69.2 \%$ of study subjects in this study are from rural areas and this might be the reason for higher proportion of intestinal obstruction incontrast to findings fom western countries and black lion hospital as acute appendicitis are common in urban area $[4,11]$.

Majority of patients in this study are in the age greater than 40 which is similar to other studies in Africa with large bowel obstruction commonly Occuring in age above 60 while small bowel cases are more common in the second and third deceds of life [14].

Male sex has stastistically significant association with intestinal obstruction than females with odd of having intestinal obstruction is 3 times more common in males than females. This my be due to large number of our patients had large bowel obstruction from sigmoid volvulus, and in our country we mostly have males who suffer from this condition. This is similar to finding from study done in Gondar, Addis Ababa, Nigeria and other western countries. The other possible reason for difference in sex might be due to the irregular bowel habits of male patients $[2,4,10,12$, 13].

Having chronic constipation has stastistically significant association with intestinal obstruction than lacking it with odd of having intestinal obstruction is 13.7 times more common in those who had chronic constipation than who lacked it. It accounts about $33.7 \%$ of intestinal obstruction cases in this study. This may be due to inadequate intake of water because of the weather condition of the area. This is similar to finding from study done in black lion hospital which comprises $33.9 \%$ but different from finding at study done in Nijeria in which constipation comprises $65 \%$. The possible reason for the difference might be due to inadequate sample size $[11,12]$.

The most commonly identified etiology of intestinal obstruction in this study is sigmoid Volvulus. From 181 study subjects $58 \%$ are caused by sigmoid volvulus. Sigmoid volvulus is also the leading cause of large bowel obstruction with $100(95.2 \%)$ of the 105 cases are caused by Sigmoid volvulus. There are similar finding to this from different parts of our country as well as other countries like sudan, Ertrea and other developing countries $[2,5,3]$ This might be due to consumption of high fiber diet of patients when compared to developed countries 'for example it accounts only for about $5-10 \%$ of causes of large bowel obstruction in USA' in which colonic tumor (colorectal cancer) is thought to be the main cause of large bowel obstruction. Small bowel volvulus in contrast to 'adhesion in black lion hospital and in developing countries' is the most common cause of Small bowel obstruction in this study. This might be due to higher proportion of abdominopelvic operation among people in developed countries and patients from urban areas previous abdominopelvic operation is clearly associated with intestinal obstruction [10]. In this study 11 of 15 patients who have previous history of abdominopelvic operation developed intestinal obstruction.

Post operative adhesion, hernia and intussusceptions are the third, fourth and fifth most common causes of intestinal obstruction with 11,12 and 8 of cases are caused by this diseases enteties. There are similar findings from recent study is Ugandan with hernia, adhesion, volvulus and intussusceptions comprising 40.2\%, 20.5\%, 17.4\% and $11.4 \%$ respectively [9].

Relatively smaller proportion $(40.3 \%)$ of patients were treated conservatively. From the total study subjects $108(59.7 \%)$ are managed by surgery. Majority $64(87.7 \%)$ of 73 conservatively treated patients are large bowel obstruction cases from sigmoid volvulus while majority 56(51.9\%) of operated cases are small bowel obstruction cases. This implies that patients with small bowel obstruction are more commonly managed by surgery than patients with large bowel obstruction. Rectal tube defilation and Nasogastric tube decompression are the two most commonly used conservative treatement methods. Failure rate of rectal tube defilation is about $11.2 \%$ only. This is similar to finding from other studies with $80-90 \%$ success rate [15].

From 108 operated cases 15(13.9\%) are diagnosed with gangrenous small intestine while 16(14.8\%) are gangrenous large intestine. This is higher than finding in other studies which demonstrated $10 \%$ strangulation rate for patients with small bowel obstruction (20). This might be due to late presentation of patients with small bowel obstruction to hospitalas $11(73.3 \%)$ of 15 patients gangrenous small obstruction presented to hospital after 48 hours of onset of illness. The reason for late 
presentation to the institution delivering thes urgical treatment needs to be studied further.

Morbidity and mortality of intestinal obstruction is related to the etiology of intestinal obstruction. Post operative complication occurred in $16.7 \%$ of operated cases. This is less than as $24.2 \%$ complication seen in Ugandan study [9]. A study at Black Lion Hospital has also got higher finding $(25.4 \%)$. The most frequently observed complication is post operative wound infection which is seen in $14(12.9 \%)$ of all operated cases. The other less frequently seen complications include anastomotic leakage in $1(0.9 \%)$, wound dehiscence in $2(1.9 \%)$, and pneumonia in one $(0.9 \%)$ of cases. This is similar to findigs from Black Lion Hospital [12].

Only $3(1.7 \%)$ of patients expired all of them were operated cases. The over all mortality rate of $1.7 \%$ from this study is less than most other studies (3-30\%), Ugandan study of $12.9 \%$ and the $15.3 \%$ mortality at Black Lion Hospital $[6,9$, $10,12]$. Adequate preoperative resuscitation and proper post operative care might be the reason for this lower mortality rate in the study area.

\section{Conclusions}

Acute intestinal obstruction remains a major cause of morbidity in our environment. Intestinal obstruction is the most common cause for non traumatic acute abdominal admission accounting $50.7 \%$ in the study area. Large bowel obstruction is relatively more common than small bowel obstruction at Debre Birhan Referral Hospital. Post operative adhesion and garoin hernias were found to be the commonest causes for intestinal obstruction next to Volvulus, but tumor was the rare cause of small bowel obstruction in the study area.

Patient in the age of 15-39 are more affected by Small bowel obstruction while age greater than 40 is highly affected by large bowel obstruction. Male patients, patients from rural areas, those with previous history of intestinal obstruction and previous history of operation are at increased risk of having intestinal obstruction.

\section{Recommendations}

\section{Debre Birhan Referral Hospital}

a, Elective repair of hernia should be strengthened before it causes obstruction.

b, Public awareness should be increased on the risk factors of intestinal obstruction through health education to reduce the observed magnitude.

c, Some patient charts were found with incomplete records should be improved.

d, Additionally every effert should be tried to decrease relapartomy rate which is associated with $0 \%$ mortality rate.

North Shoa Zonal Health Department and DBRH

The knowledge gap of mid and lower level health professionals on the diagnosis, resuscitation and importance of early referral to higher center should be addressed.

Moreover, health facilities and health professionals capable of handling patients with intestinal obstruction should be available with in the reach of the community.

\section{References}

[1] MichaelJ.Zinner, Stanley W. Ashley et al: Maingot's Abdominal Operations. 11th Ed. New York: McGraw Hill, 2007.

[2] Craig T. Albanese, John T. Anderson et al; Current surgical diagnosis and treatment .12th ed. California: The McGrawHill Companies, Inc, 2006.

[3] F. Charles Brunicardi, Dana K. Andersen et al: Schwartz Principles of Surgery. $9^{\text {th }}$ Ed. Texas: McGraw-Hill, 2010.

[4] Zeki Abdurahman, Mohammed Kedir.Chronic Sigmoid VolvulusEthiop. J. Health Biomed Sci., 2010. Vol.3, No.1.

[5] Kendrick ML. Partial small bowel obstruction: clinical issues and recent technical advances. Abdom Imaging 2009; 34:329.

[6] Shawana Asad, Hafizullah Khan et al; aetiological factors in mechanical intestinal obstruction. J Ayub Med Coll Abbottabad. 2011; 23(3).

[7] Kotiso, B., Abdurahman, Z.: Pattern of acute abdomen in adult patients in Tikur Anbessa Teaching hospital, Addis Ababa, Ethiopia; East and Central African Journal of Surgery, April, 2006 .12( 1), 47-52.

[8] Abeje w et al.: Retrospective analysis of mortalities in a tertiary care hospital in Northeast Ethiopia.BMC Research Notes20147:46.

[9] COSECSA/ASEA Publication- Inguinal Hernia Repair is Safe in Africa-East and Central African Journalof Surgery. July/August; 2013 Volume 18 (2).

[10] P. K Okeny, T. G Hwang, D. M Ogwang: Acute Bowel Obstruction in a Rural Hospitals in Northern Uganda.East and Central African Journal of Surgery Volume 16 Number 1 March/April 2011.

[11] Steven h. Untracht. Surgary in Africa, where resources are scarce: optimal management of intestinal obstruction and similar abdominal emergencies. Johnstown, Pennsylvania, USA.1, 1-30.

[12] B. Kotiso, Z. Abdurrahman. Pattern of Acute Abdomen in Adult Patients in Tikur Anbessa Teaching Hospital, Addis Ababa, Ethiopia. East and Central African Journal of Surgery. April 2007.12(1), 47-52.

[13] Lawal, O. O., Olayinka, O. S. and Bankole, J. O. Spectrum of causes of intestinal obstruction in adult Nigerian patients. 2005, S. African J. Surg., 43:34-36.

[14] Ohene-Yeboah, M. Acute surgical admissions for abdominal pain in adults in Kumari, Ghana. 2006, Aust. N. Zealand J. Surg., 76:898-903.

[15] GEORGE D. ZUIDEMA, CHARLES J. YEO et al, Shackelford's Surgery of the Alimentary Tract, 5th Ed, Ann Arbor, Michigan: W. B. Saunders Company, 2002. 